\title{
Numerical modelling of evaporation in a ceramic layer in the tape casting process
}

\author{
Jabbaribehnam, Mirmasoud; Jambhekar, V. A.; Hattel, Jesper Henri; Helmig, R.
}

\section{Published in:}

A I P Conference Proceedings Series

Link to article, DOI:

$10.1063 / 1.4951796$

Publication date:

2016

Document Version

Publisher's PDF, also known as Version of record

Link back to DTU Orbit

Citation (APA):

Jabbaribehnam, M., Jambhekar, V. A., Hattel, J. H., \& Helmig, R. (2016). Numerical modelling of evaporation in a ceramic layer in the tape casting process. A I P Conference Proceedings Series, 1738, [030040].

https://doi.org/10.1063/1.4951796

\section{General rights}

Copyright and moral rights for the publications made accessible in the public portal are retained by the authors and/or other copyright owners and it is a condition of accessing publications that users recognise and abide by the legal requirements associated with these rights.

- Users may download and print one copy of any publication from the public portal for the purpose of private study or research.

- You may not further distribute the material or use it for any profit-making activity or commercial gain

- You may freely distribute the URL identifying the publication in the public portal

If you believe that this document breaches copyright please contact us providing details, and we will remove access to the work immediately and investigate your claim. 


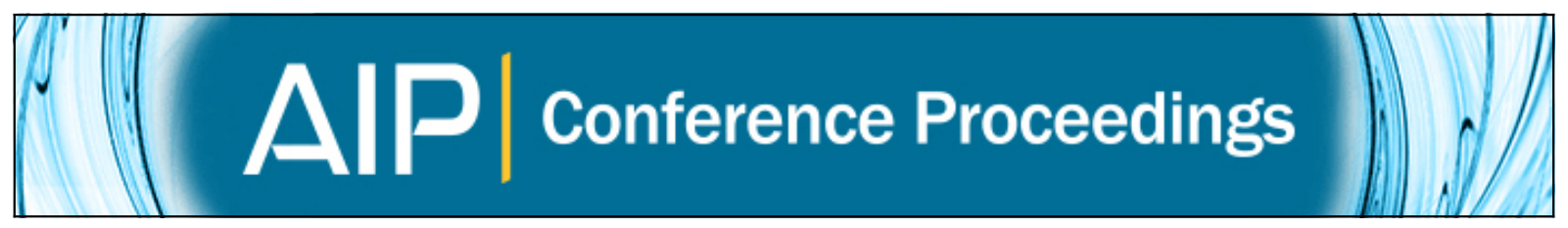

Numerical modelling of evaporation in a ceramic layer in the tape casting process

M. Jabbari, V. A. Jambhekar, J. H. Hattel, and R. Helmig

Citation: AIP Conference Proceedings 1738, 030040 (2016); doi: 10.1063/1.4951796

View online: http://dx.doi.org/10.1063/1.4951796

View Table of Contents: http://scitation.aip.org/content/aip/proceeding/aipcp/1738?ver=pdfcov

Published by the AIP Publishing

Articles you may be interested in

Process modelings and simulations of heavy castings and forgings

AIP Conf. Proc. 1532, 81 (2013); 10.1063/1.4806811

Numerical Modeling of Fluid Flow in the Tape Casting Process

AIP Conf. Proc. 1389, 143 (2011); 10.1063/1.3636690

A Numerical Model of the Temperature Field of the Cast and Solidified Ceramic Material

AIP Conf. Proc. 1252, 571 (2010); 10.1063/1.3457605

3D Numerical modeling of thermomechanical processes during continuous casting for shape rolling AIP Conf. Proc. 712, 1265 (2004); 10.1063/1.1766703

Application of process modeling to improve the competitiveness of investment castings

AIP Conf. Proc. 361, 89 (1996); 10.1063/1.49984 


\title{
Numerical Modelling of Evaporation in A Ceramic Layer in the Tape Casting Process
}

\author{
M. Jabbari*, V.A. Jambhekar ${ }^{\dagger}$, J.H. Hattel* and R. Helmig ${ }^{\dagger}$ \\ *Process Modelling Group, Department of Mechanical Engineering, Technical University of Denmark, Nils \\ Koppels Allé, 2800 Kgs. Lyngby, Denmark. \\ ${ }^{\dagger}$ Department of Hydromechanics and Modelling of Hydrosystems, Institute for Modelling Hydraulic and \\ Environmental Systems, Universität Stuttgart, Stuttgart, Germany.
}

\begin{abstract}
Evaporation of water from a ceramic layer is a key phenomenon in the drying process for the manufacturing of tape cast ceramics. This process contains mass, momentum and energy exchange between the porous medium and the freeflow region. In order to analyze such interaction processes, a Representative Elementary Volume (REV)-scale model concept is presented for coupling non-isothermal multi-phase compositional porous-media flow and single-phase compositional laminar free-flow. The preliminary results show the typical expected evaporation behaviour from a porous medium initially saturated with water, and its transport to the free-flow region according to the existent results from the literature.
\end{abstract}

Keywords: Evaporation, ceramics, free-flow, porous-media, coupling.

PACS: $81.05 . J e, 02.60 . \mathrm{Lj}, 47.56 .+\mathrm{r}, 83.10 .-\mathrm{y}$

\section{INTRODUCTION}

Being mainly used in the electronics industry as a forming method for ceramics, tape casting is increasingly used in the production of numerous multilayer applications and electronic substrates, like e.g. capacitors, piezoelectric actuators, gas sensors, etc., where high quality and low geometry tolerances are required $[1,2]$. In the tape casting process, the ceramic slurry is mostly categorized as a non-Newtonian fluid with relatively high viscosity $[2,3,4,5]$. A summary of work published regarding the rheological classification of non-Newtonian fluids and the existence of analytical/numerical models with focus on tape casting has been given previously by Jabbari et al. [2].

Tape casting consists of three major sub-processes which are: (1) tape casting of an aqueous (fluid) ceramic slurry in a doctor blade configuration, (2) drying of the green tape, and (3) additional processing (which is often a sintering process). The first and third stages have been modelled extensively with numerical methods $[2,3,4,5,6]$ whereas the second has remained almost unexplored numerically. An example of simple numerical simulation for the evaporation of water based ceramics was first developed by Jabbari and Hattel [7], using a semi-coupled heat and mass transfer model.

A tape layer can be considered as a porous medium which contains powders and liquid phases [8]. The prediction of evaporative drying rates from porous-media remains a challenge due to the ambient conditions at the interface (radiation, humidity, temperature, air velocity, turbulent conditions) $[9,10,11]$. Moreover, the internal porousmedium properties lead to abrupt transitions and rich flux dynamics. The factors involved are coupled by the complex interactions between the porous medium and the free-flow system.

Modelling such coupled systems while accounting for the respective processes in both domains is a challenging task, especially since many of these systems are dominated by multi-phase compositional flow [9,10,11]. Mosthaf et al. [12] developed a coupling concept for non-isothermal two-phase compositional flow in a porous medium in contact with a laminar single-phase non-isothermal compositional system in the free-flow region. It is based on existing approaches and valid on the Representative Elementary Volume (REV) scale. The employed coupling conditions for mass, momentum and energy account for the physics at the interface and are based on flux continuity and thermodynamic equilibrium and justified by phenomenological explanations. The resulting coupled model is flexible with respect to the sub-domain models which are combined by clearly defined coupling conditions. It allows a detailed description of transfer processes like, e.g. evaporation influenced by a laminar wind field.

The focus of this paper is to simulate the evaporation phenomenon in the drying of a thin ceramic layer like in e.g. tape casting based on the models developed by Mosthaf et al. [12]. In the following, the developed model concept consisting of sub-domain models and coupling conditions will be briefly explained, and a brief overview on the 
numerical concept and on the implementation of the coupled model into the modelling toolbox $\mathrm{DuMu}^{x}$ is given [13].

\section{CONCEPTUAL MODEL}

Figure 1(a) shows the model concept in which two-phase flow (gas and liquid) in the porous medium, and a single phase (gas phase) in the free-flow region are assumed. A compositional (miscible) model is considered where each phase consists of two components (air and water). In the gas phase, water is present in the form of vapor. The liquid phase contains dissolved air. The gas mixture air is approximated as one component, assuming that nitrogen and oxygen have similar properties and that all other constituents are negligible. The following processes are to be described: transfer of heat and vapour across the interface, evaporation and condensation at the interface, vaporization/ condensation, and dissolution/degassing inside the porous medium.

To simplify the system in the free-flow region, $\Omega^{\text {ff }}$, we assume slow flow conditions, neglect the nonlinear inertia forces, and consider unsteady Stokes flow. Neglecting the inertia term is a simplification that is done in order to explain the coupling concept on the basis of a comparatively simple model. In the porous medium, $\Omega^{\mathrm{pm}}$, the multi-phase Darcy law in combination with a mass balance equation for a component, the total mass balance and an energy balance are used, see, for example, Class et al. [14]. Moreover, we assume local thermodynamic equilibrium to hold and all fluids to be Newtonian. For simplicity of notation, the superscripts (ff) and (pm) are only applied for the quantities at the interface, where (ff) refers to the values in the free-flow sub-domain and (pm) stands for the porous-medium side.

Here, the coupling of the two domains is achieved using the two-domain approach. The interface is assumed to be simple in the sense that it cannot store mass, momentum or energy, and assumes continuity of fluxes and local thermodynamic equilibrium [15]. Furthermore, the Beavers-Joseph-Saffman condition is employed in the knowledge of its theoretical limitation to single-phase, parallel flow [16, 17].

The problem setting is illustrated in Figure 1(b) two domains $\Omega^{\mathrm{ff}}$ and $\Omega^{\mathrm{pm}}$ are separated by the interface $\Gamma=$ $\partial \Omega^{\mathrm{ff}} \cap \partial \Omega^{\mathrm{pm}}$ with the outward unit normal vectors $\mathbf{n}^{\mathrm{ff}}$ and $\mathbf{n}^{\mathrm{pm}}$.
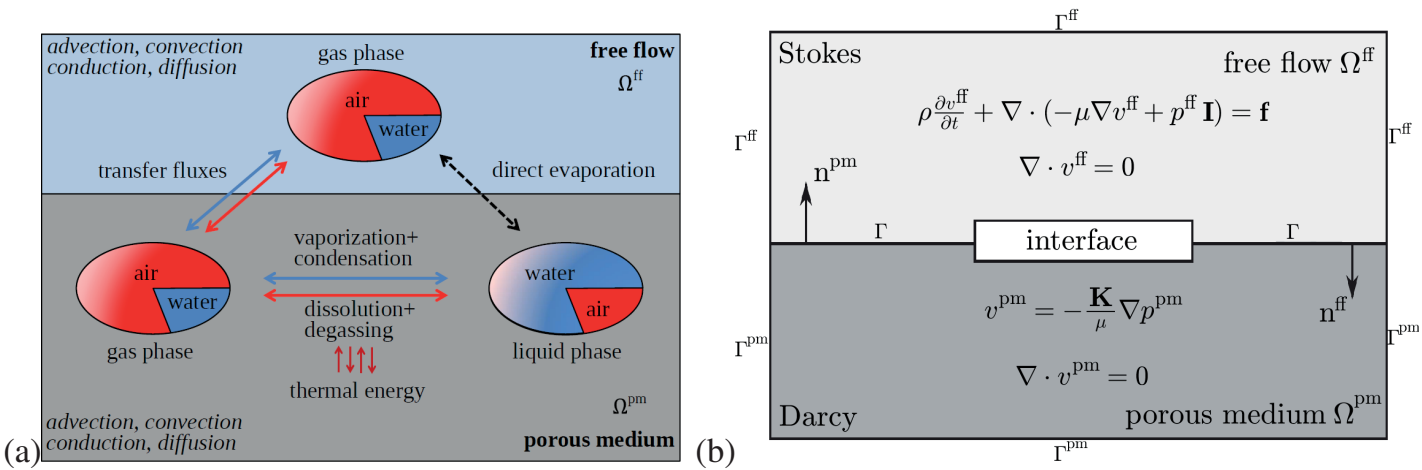

FIGURE 1. Model concept with a single phase in the free-flow that interacts with two fluid phases in the porous medium.

\section{NUMERICAL MODELLING}

The control-volume finite-element method (CVFEM or box method) is used for the discretization of both subdomains $\Omega^{\mathrm{ff}}$ and $\Omega^{\mathrm{pm}}[18,19]$ together with an implicit Euler time discretization. Full details of the discretization scheme, its definition as well as the stabilization technique to the continuity equation are given in depth by Baber et al. [9].

The pressure of the gas phase $p_{\mathrm{g}}$, the saturation of the liquid phase $S_{l}$ and the temperature $T$ are chosen to be the primary variables in the porous medium $\Omega^{\mathrm{pm}}$. However, due to appearance and disappearance of the liquid phase, a switch of primary-variable [14] has been used similar to that from Baber et al. [9]. In the free-flow domain $\Omega^{\mathrm{ff}}$, the pressure of the gas phase $p_{\mathrm{g}}$, the mass fraction of water in the gas phase $X_{\mathrm{g}}^{w}$, the velocity of the gas phase $\mathbf{v}_{\mathrm{g}}$ and the temperature $T$ are chosen as primary variables.

The implementation of the numerical scheme has been conducted in the modelling toolbox DuMu${ }^{x}$, which is a free and open-source simulator for flow and transport processes in porous-media, based on the Distributed and Unified 
Numerics Environment (DUNE) [20]. The coupled problem (free-flow domain, porous-medium domain and interface) is written in the following operator form

$$
\frac{\partial \mathbf{M}(\mathbf{u})}{\partial t}-\nabla \cdot \mathbf{F}(\mathbf{u})=\mathbf{Q}(\mathbf{u})
$$

where $\mathbf{u}=\left(p_{\mathrm{g}}^{\mathrm{ff}}, X_{\mathrm{g}}^{w, \mathrm{ff}}, \mathbf{v}_{\mathrm{g}}^{\mathrm{ff}}, T^{\mathrm{ff}}, p_{\mathrm{g}}^{\mathrm{pm}}, S_{l}^{\mathrm{pm}}\right.$ or $\left.X_{\mathrm{g}}^{w, \mathrm{pm}}, T^{\mathrm{pm}}\right)$ is the solution vector, $\mathbf{M}(\mathbf{u})$ is the storage, $\mathbf{F}(\mathbf{u})$ the flux and $\mathbf{Q}(\mathbf{u})$ the source/sink term.

The nonlinear algebraic system at each time step is treated via a standard Newton solver, which is a stable and robust scheme, as follows:

$$
\underbrace{\left(\frac{\partial \mathbf{R}}{\partial \mathbf{u}}\right)_{n+1, m}}_{\mathbf{J}\left(\mathbf{u}^{n+1, m}\right)} \underbrace{\left(\mathbf{u}^{n+1, m}-\mathbf{u}^{n+1, m-1}\right)}_{\Delta \mathbf{u}}=-\mathbf{R}\left(\mathbf{u}^{n+1, m}\right)
$$

where $\mathbf{J}\left(\mathbf{u}^{n+1, m}\right)$ is the Jacobi matrix calculated by numerical differentiation, $\Delta \mathbf{u}$ is the correction to the primary variables $\mathbf{u}$, and $\mathbf{R}\left(\mathbf{u}^{n+1, m}\right)$ is the residuum at time level $n+1$ and iteration $m$. The linear problem at each Newton iteration step is solved using the direct linear solver SuperLU [21], and for the time integration, a fully implicit Euler scheme with a heuristic time-step control based on the convergence rate of the Newton solver is used $[9,10,11,12,22]$.

\section{RESULTS}

A two-dimensional domain is chosen for simulating the evaporation, which is illustrated in Figure 2. The tape layer with the dimension of $x \times y=0.01 \mathrm{~m} \times 0.005 \mathrm{~m}$ is assumed to be a fully water-saturated porous medium that is closed on all other sides with a relatively dry air blowing horizontally over the surface $\left(y^{\text {interface }}=0.005 m\right.$ ). All boundary types are also shown in Figure 2, and as seen a graded mesh is employed that becomes finer towards the interface. In this study the gravitational force $(\mathbf{g})$ is neglected in the free-flow region, and for the porous domain an isotropic permeability $\left(\mathbf{K}=3 \times 10^{-10}\right)$ with a constant porosity $(\phi=0.4)$ is assumed. The initial values of $p_{\mathrm{g}}^{\mathrm{ff}}=p_{\mathrm{g}}^{\mathrm{pm}}=1 \times 10^{5}, X_{\mathrm{g}}^{w, \mathrm{ff}}=0.008, T^{\mathrm{ff}}=T^{\mathrm{pm}}=298.15 K$, and $S_{w}^{\mathrm{pm}}=0.98$

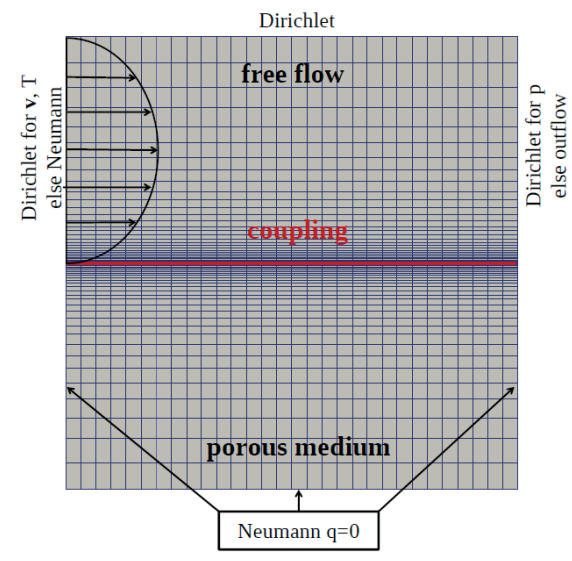

FIGURE 2. Model setup and grid structure.

A parabolic velocity profile with a maximum velocity of $v_{\max }$ is chosen in the left boundary for the free-flow, $\mathbf{v}_{x}=$ $\frac{4 \times v_{\max }}{\left(y_{\max }^{\mathrm{ff}}-y_{\min }\right)^{\mathrm{ff}}}\left(y-y_{\min }^{\mathrm{ff}}\right)\left(y-y_{\max }^{\mathrm{ff}}\right)$, and here for the reference case $v_{\max }=0.1 \mathrm{~m} / \mathrm{s}, y_{\max }^{\mathrm{ff}}=0.01 \mathrm{~m}$, and $y_{\min }^{\mathrm{ff}}=0.005 \mathrm{~m}$.

Numerical results of spatial and temporal evolution for the saturation of water in the porous-media $\left(S_{w}\right)$ as well as mass fraction of vapour in the gas phase $\left(X_{\mathrm{g}}^{w, f f}\right)$ are shown in Figure 3. The results are in a good agreement with the ones presented in the similar studies $[9,10,11,12,22]$. As seen from Figure 3, during the evaporation process, the saturation of the water in the porous medium is decreasing by time. Hence, the mass fraction of vapour in the free-flow (especially in the interface region) is increased. However, at later times in the process the mass fraction of vapour in the free-flow is decreased, since the porous region is desaturated already. 
(a)

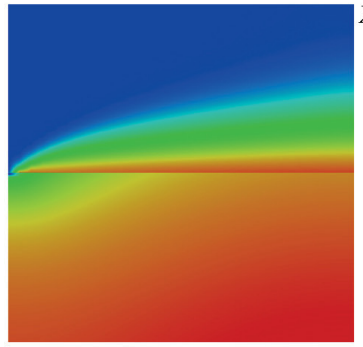

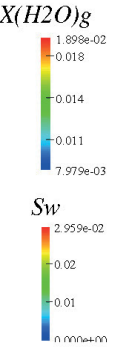

(b)

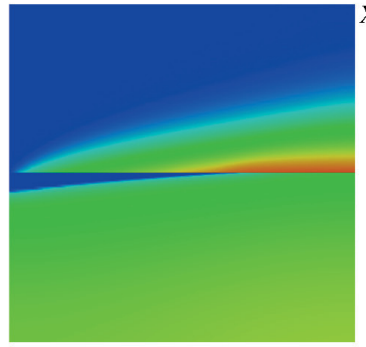

(c)

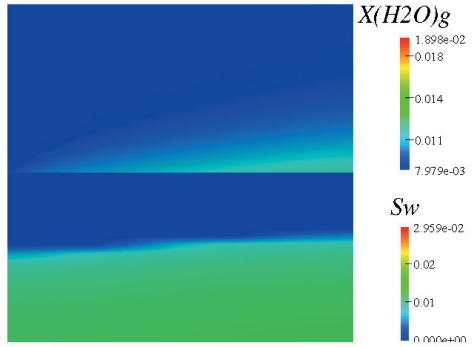

FIGURE 3. Evolution of water saturation in the porous-media and mass fraction of vapour in the free-flow region after (a) 7 [min], (b) 27 [min], and (c) 43 [min].

\section{CONCLUSIONS}

In this work a fully coupled model to describe the interaction between the porous-media and the free-flow for evaporation-driven transport in drying of a ceramic layer is presented. The model concept is based on the two-domain approach, where a non-isothermal two-phase compositional model is applied in the porous-media sub-domain and single-phase compositional model is used in the free-flow sub-domain. A simple interface is employed which cannot store mass, momentum and energy. The coupling conditions accounts for mass, momentum and energy exchange between the sub-domains and assures continuity of fluxes. The model is implemented in the open source modelling framework DuMux [13]. The model depicts the evaporation process well [7] and it is in a good agreement with the ones presented in the similar studies $[9,10,11,12,22]$.

\section{REFERENCES}

1. M. Jabbari, and J. Hattel, "Numerical modeling of fluid flow in the tape casting process," in ICNAAM-2011, edited by T. E. Simos, and C. Tsitouras, AIP Conference Proceedings 1, American Institute of Physics, New York, 2011, pp. 143-146.

2. M. Jabbari, R. Bulatova, J. H. Hattel, and C. R. H. Bahl, Mater. Sci. Tech. 29, 1080-1088 (2013).

3. M. Jabbari, and J. H. Hattel, J. Am. Ceram. Soc. 96, 1414-1420 (2013).

4. M. Jabbari, and J. H. Hattel, Mater. Sci. Tech. 30, 283-288 (2014).

5. M. Jabbari, R. Bulatova, J. H. Hattel, and C. R. H. Bahl, App. Math. Modell. 38, 3222-3232 (2014).

6. E. A. Olevsky, Mater. Sci. Eng. R 23, 41-100 (1998).

7. M. Jabbari, and J. Hattel, Drying Technol. In Press (2015).

8. C. Bauer, M. Cima, A. Dellert, and A. Roosen, J. Am. Ceram. Soc. 92, 1178-1185 (2009).

9. K. Baber, K. Mosthaf, B. Flemisch, R. Helmig, S. Müthing, and B. Wohlmuth, IMA J. App. Math. hxs048, 1-23 (2012).

10. V. Jambhekar, N. schröder, R. Helmig, and N. Shokri, Transport Porous Med. Accepted (2015).

11. V. Jambhekar, N. schröder, E. Mejri, R. Helmig, and N. Shokri, Transport Porous Med. Submitted (2015).

12. K. Mosthaf, K. Baber, B. Flemisch, R. Helmig, A. Leijnse, I. Rybak, and B. Wohlmuth, Water Resour. Res. 47, 1-19 (2011).

13. B. Flemisch, M. Darcis, K. Erbertseder, B. Faigle, A. Lauser, K. Mosthaf, S. Müthing, P. Nuske, A. Tatomir, M. Wolff, and R. Helmig, Adv. Water Res. 25, 1102-1112 (2002).

14. H. Class, R. Helmig, and P. Bastian, Adv. Water Res. 34, 533-550 (2011).

15. S. Hassanizadeh, and W. Gray, Water Resour. Res. 25, 529-539 (1989).

16. G. S. Beavers, and D. D. Joseph, J. Fluid Mech. 30, 197-207 (1967).

17. R. Saffman, Stud. App. Math. 50, 93-101 (1971).

18. C. Masson, H. J. Saabas, and B. R. Baliga, Int. J. Num. Methods Fluids 18, 1-26 (1994).

19. R. Huber, and R. Helmig, Comput. Geo. 14, 141-164 (2000).

20. P. Bastian, M. Blatt, A. Dedner, C. Engwer, R. Klöfkorn, R. Kornhuber, M. Ohlberger, and O. Sander, Computing 82, 121-138 (2008).

21. J. W. Demmel, S. C. Eisenstat, J. R. Gilbert, X. S. Li, and J. W. H. Liu, SIAM J. Mat. Ana. Appl. 20, $720-755$ (1999).

22. K. Mosthaf, R. Helmig, and D. Or, Water Resour. Res. 50, 1059-1079 (2014). 